\title{
Human Pacman: A Mobile Entertainment System with Ubiquitous Computing and Tangible Interaction over a Wide Outdoor Area
}

\author{
Adrian David Cheok, Siew Wan Fong, Kok Hwee Goh, Xubo Yang, Wei Liu, \\ Farzam Farzbiz, and Yu Li \\ National University of Singapore \\ \{adriancheok, engp1620,g0202687, eleyxb, eleliuw, eleff, elely\}@nus.edu.sg
}

\begin{abstract}
Human Pacman is an interactive role-playing game that envisions to bring the computer gaming experience to a new level of emotional and sensory gratification by setting the real world as a playground. This is a physical fantasy game integrated with human-social and mobile-gaming that emphasizes on collaboration and competition between players. By setting the game in a wide outdoor area, natural human-physical movements have become an integral part of the game. Pacmen and Ghosts are now human players in the real world experiencing mixed reality visualization from the wearable computers on them. Virtual cookies and actual physical objects are incorporated to provide novel experiences of seamless transitions between real and virtual worlds and tangible human computer interface respectively. We believe Human Pacman is pioneering a new form of gaming that anchors on physicality, mobility, social interaction, and ubiquitous computing.
\end{abstract}

\section{Introduction}

Human Pacman is a real-world-physical, social, and wide area mobile entertainment system that is built upon the concepts of ubiquitous computing, tangible human-computer interaction, and wide-area entertainment networks. The game has several novel aspects: Firstly, the players immersively role-play the characters of the Pacmen and Ghosts in the real physical world. Secondly, users enjoy unrestricted movement outdoor and indoor while maintaining their social contacts. Thirdly, Human Pacman also explores novel tangible aspects of human physical movement and perception, both on the player's environment and on the interaction with the digital world.

In this system the users are provided with custom-built wearable computers; and they interact both directly with other players when in physical proximity, or indirectly via the Wireless LAN network. Virtual fantasy and imaginative play activity elements, which have made computer game popular [1], are incorporated using augmented reality techniques. The players also experience seamless transitions between real and virtual worlds as they swap between immersive first person augmented reality view and full virtual reality view of the Pac-world throughout the game.

L. Chittaro (Ed.): Mobile HCI 2003, LNCS 2795, pp. 209-224, 2003.

(C) Springer-Verlag Berlin Heidelberg 2003 
Employing the philosophy of ubiquitous computing [2], we have implemented a system that embeds everyday physical objects with digital fantasy meanings. For example, we have attached Bluetooth devices to sugar jars which when being picked up, will automatically communicate with the wearable computer by adding the corresponding virtual ingredient to the inventory list of the player.

Each of the novel interactions of Human Pacman mentioned is summarized in Table 1. We will proceed by firstly giving a research background to this system and previous works that have motivated us. Then we go on to clarify the actual game play designed. More importantly in the section that follows, the system is analyzed in the context of addressing various Human Computer Interaction issues. Lastly we conclude with our reflections on the future impacts of the system on everyday life.

\section{Background}

Entertainment with interactivity [3] and sociality [4] are becoming important as reflected in the growing popularity of online games [4]. These computer games provides unrivalled richness of human interaction [5] between players despite of geographical separation. Nevertheless, social interaction between players is limited with no behavioural engagement and cognitive exchange. Human Pacman aims to merge interactive aspects of networked gaming with the real physical world, while maintaining social contact in reality and virtuality.

Human Pacman has aspects derived from pioneering work on ubiquitous gaming. Multi-players mobile gaming is demonstrated in 'Pirates!' [6] which is a game on PDAs with proximity sensing technology. However, it provides little immersive experience since there is no Augmented Reality and Virtual Reality support. The E3 project [7] examines the essential elements of free play, and multi-user social interaction. However it does not explore large-scale configuration where users walk around.

Augmented reality techniques are used as part of Human Pacman's interface. Previous works done on using augmented reality in entertainment include AR2 Hockey [8] and AquaGaunlet [9]. However, these games are played in a small area, with limited movement and little interaction with physical space. Another important mobile game is known as ARQuake [10], which is an AR extension of the popular computer game Quake. Using Wearable Computer equipped with global positioning system, ARQuake can be played indoor and outdoor. However it is a single player game with practically no social interaction.

Lastly, the transition between the real and physical world in Human Pacman is derived from research that has been done on continual transversal along the Reality-Virtuality continuum [11]. The Magic Book [12] uses a book metaphor to demonstrate the seamless transitions between augmented and virtual reality. Nevertheless collaboration is carried out only in a small-scale and closed-up configuration. Touch-Space [13] is an embodied computing based mixed reality game space with free movement between the real world and virtual world. However they are constrained by small game space and limited physical movement. 
Table 1. Detail descriptions of each novel features of Human Pacman.

\begin{tabular}{|c|c|}
\hline Feature & Details \\
\hline Physical Gaming & $\begin{array}{l}\text { Players are physically role-playing the characters of Pac- } \\
\text { men and Ghost; with Wearable Computers donned, they } \\
\text { use free bodily movements as part of interaction between } \\
\text { each person, and among objects in the real wide area } \\
\text { landscapes and virtual environments. }\end{array}$ \\
\hline Social Gaming & $\begin{array}{l}\text { Players interact both directly with other players when } \\
\text { they are in physical proximity, or indirectly via the Wire- } \\
\text { less LAN network by instant messaging. All Internet } \\
\text { users can participate in the game by viewing and col- } \\
\text { laborating with real Human Pacmen and Ghosts. }\end{array}$ \\
\hline Mobile Gaming & $\begin{array}{l}\text { Players are free to move about in the indoor } \backslash \text { outdoor } \\
\text { space without being constrained to the } 2 \mathrm{D} \backslash 3 \mathrm{D} \text { screen of } \\
\text { desktop computers. }\end{array}$ \\
\hline $\begin{array}{l}\text { Ubiquitous Comput- } \\
\text { ing }\end{array}$ & $\begin{array}{l}\text { Everyday objects throughout the environment seamlessly } \\
\text { have a real-time fantasy digital world link and mean- } \\
\text { ing. There is automatic communication between Wear- } \\
\text { able Computers and Bluetooth devices embedded in cer- } \\
\text { tain physical objects used in game play. }\end{array}$ \\
\hline Tangible Interaction & $\begin{array}{l}\text { Throughout the game people interact in a touch and tan- } \\
\text { gible manner. For example, Players need to physically } \\
\text { pick up objects and tap on the shoulder of other players } \\
\text { to devour them. }\end{array}$ \\
\hline $\begin{array}{l}\text { Outdoor Wide-Area } \\
\text { Gaming Arena }\end{array}$ & $\begin{array}{l}\text { Large outdoor areas can be set up for the game whereby } \\
\text { players carry out their respective missions for the role } \\
\text { they play. }\end{array}$ \\
\hline $\begin{array}{l}\text { Seamless Transition } \\
\text { between real and vir- } \\
\text { tual worlds }\end{array}$ & $\begin{array}{l}\text { Players swap freely between immersive first person aug- } \\
\text { mented reality view and full virtual reality view of the } \\
\text { Pac-world in the game. }\end{array}$ \\
\hline
\end{tabular}

In Human Pacman, the interface and transition between the real world and virtual world is achieved in real time throughout the spacious indoor and outdoor physical world.

\section{System Design and Game Play}

Human Pacman features a centralized architecture that is made up of four main entities, namely the central server, wearable computers, laptops, and Bluetooth embedded objects. An overview of the system is shown in the Fig. 1.

The underlying program is built on a client server architecture with wearable computers and helper laptops as clients, and the desktop computer as a cen- 


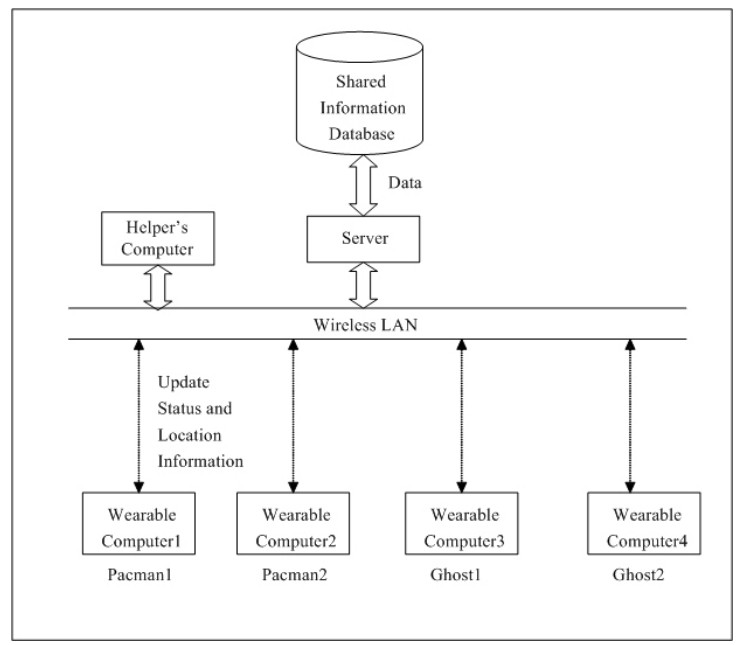

Fig. 1. Complete top level system design overview of Human Pacman.

tral server communicating via Wireless LAN. Physical location and players' status updates are done between the client wearable computers and the server on a regular basis. The server maintains up-to-the-minute players' information, and presides over any communication between Bluetooth objects and the wearable computers. Detail configuration of the wearable computers as seen in Fig. 2 is described as follows.

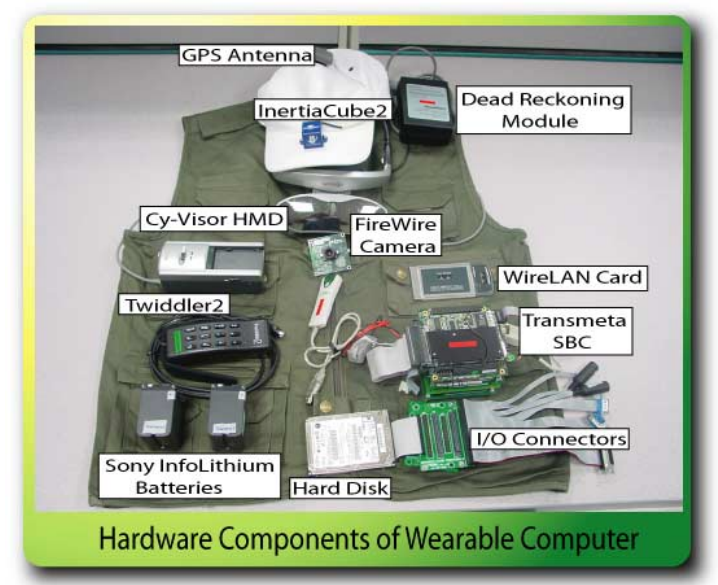

Fig. 2. Detail configuration of wearable computer 
The main components of the wearable computers are Transmeta Single Board Computer (PC104 plus compliant motherboard running on Crusoe processor), Twiddler2 (handheld keyboard and mouse), Cy-Visor Head Mounted Display (video see-through HMD) with FireWire camera attached, two Sony F960 InfoLithium batteries, InertiaCube2 (inertia sensor from Intersense [14]), DRMIII module (GPS and Dead-Reckoning device from Point Research Corporation [15]), and JVC Bluetooth device. Position tracking is done using DRM which measures the displacement of the user from an initialization point by measuring the direction (with data obtained from the compass), and distance traveled (using accelerometer data) with each footstep taken. Although the DRM is a self-contained navigation unit, in-built GPS receiver can be used to collect data for the correction of both the distance and direction calculations. InertiaCube2, which is used for head tracking for the implementation of augmented reality display, is an inertial three degree-of-freedom orientation tracking system of high accuracy.

With the software architecture mentioned as the backbone of the game engine and the hardware as enabling tools, we proceed to describe the game play of Human Pacman and then discuss some of the problems we have encountered.

\subsection{Main Concepts: Team Collaboration, Ultimate Game Objectives and the Nature of Pac-World}

The players are assigned to two opposing teams, namely the Pacman team and the Ghost team. Each Pacman $\backslash$ Ghost is in coalition with one Helper who is an Internet online player.

Human Pacman has similar game objectives as the original Pacman. Basically the goal of the Pacman team is to collect all virtual plain cookies in Pac-World while avoiding the Ghosts. Meanwhile, the aim of the Ghost team is to devour all Pacmen. For excitement of game play, after 'eating' special ingredients, a Pacman gains Ghost-devouring capability and henceforth can attack her enemy for a limited period of time.

Pac-World is a fantasy world existing dualistically in both Augmented Reality (AR) and Virtual Reality (VR) mode. Pacmen and Ghosts are allowed to switch between the two viewing modes; whereas Helpers can only view in VR mode. Most importantly there is a real time link between the wide-area physical world and the virtual Pac-World, thus providing the users with a marriage of fantasy digital world and realistic physical world. As seen in Fig. 3 where the 2D map of the selected game play area in our university campus and the 3D map of Pac-World are shown side-by-side, we have converted the real world to a fantasy virtual playground by ingraining the latter with direct physical correspondences.

\subsection{Pacman, Ghost, and Helper}

Pacman has to physically move about collecting virtual cookies in AR mode where the real world is being overlaid with them as shown in Fig. 4. In addition 


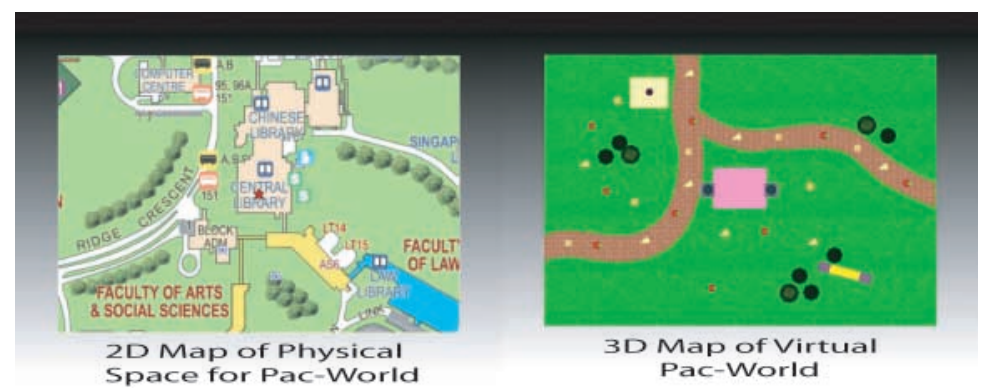

Fig. 3. 2D map of game play area and its corresponding 3D map of Pac-world

to the virtual cookies, she has to find and collect physical ingredients as shown in Fig. 5. Besides, the Pacman should avoid the Ghost, i.e. not letting Ghost tapping on her shoulder capacitive sensor pad. This physical touch interaction exemplifies tangible physical interaction between humans, which is commonly found in traditional games such as hide-and-seek, but is now being revived in computer gaming arena. The role of a Ghost is simply to track down all Pacmen and devour them. Nevertheless, she has to shun of Pacmen with Ghost-devouring power. Helper is a new character in Human Pacman who acts as an advisor to her partner(a Ghost or Pacman), informing her the positions of enemy mobile units and ingredients which are not labelled in AR mode. Therefore the Helper messages important information to her partner as shown in Fig. 6 and thus this promotes collaboration and interaction between human.

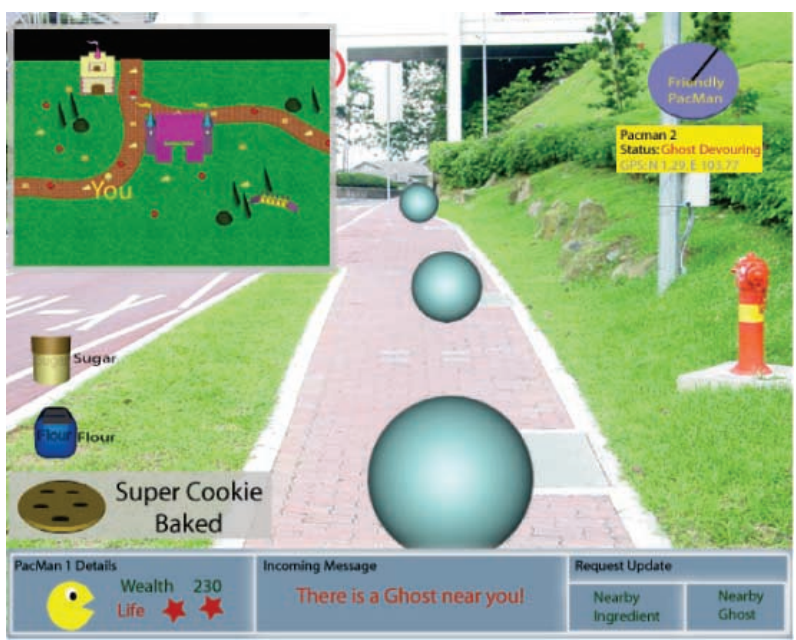

Fig. 4. First person view as presented in HMD display of Pacman

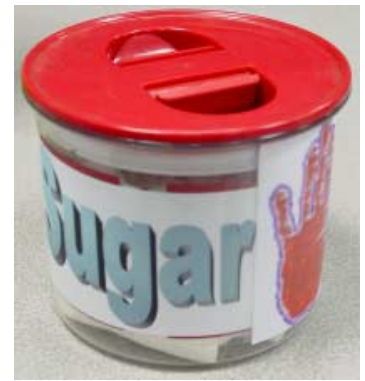

Fig. 5. Bluetooth embedded object 


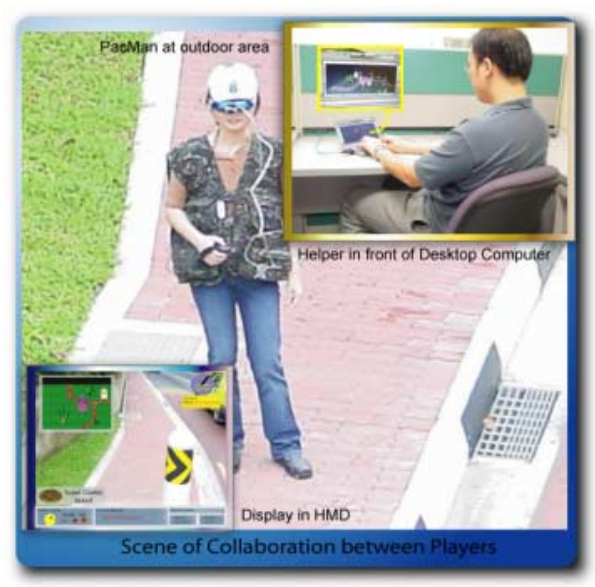

Fig. 6. Close collaboration between the outdoor Pacman and her Helper

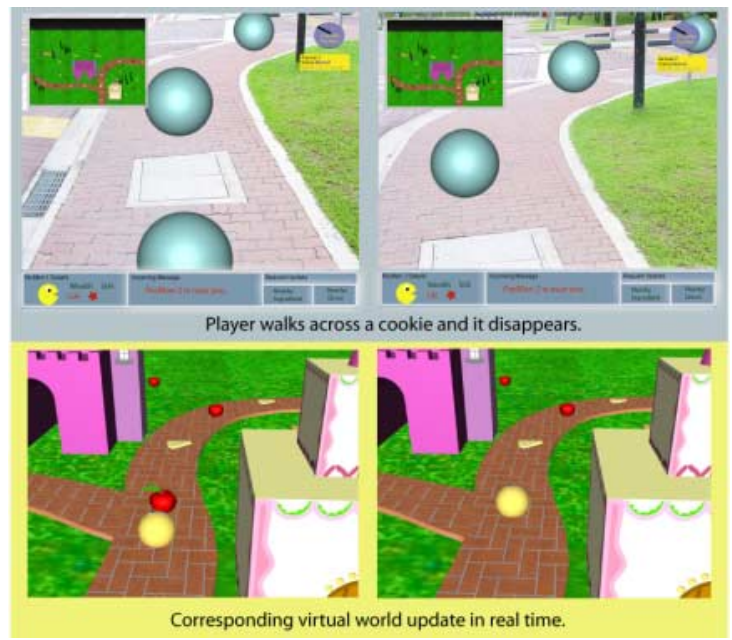

Fig. 7. Pacman collecting cookies

\subsection{Actual Game Play}

Starting the Game: Pacmen and Ghosts start from two different physical locations called Pac-castle and Ghost-house in Pac-world (physical game area).

Collection of Plain Cookies: When Pacman collects a cookie by walking through it, this action is reflected visually in Pac-World through the disappearing of the cookie in both the AR and VR mode as shown in Fig. 7. 


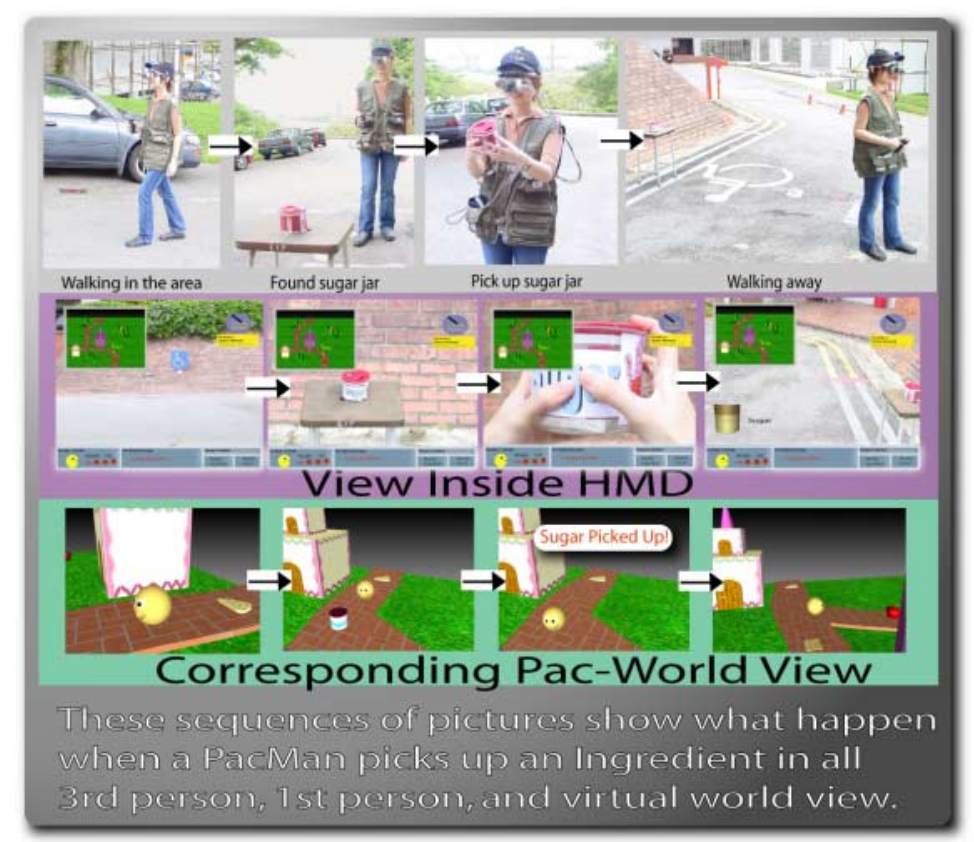

Fig. 8. Sequence of pictures showing the collection of an ingredient

Collection of Ingredients: In the game, Pacman collects ingredients including flour, butter, sugar, and special ingredients (e.g. Chocolate Chip, Almond) to make special cookies. There are two types of them: a butter cookie is made up of flour, butter, and sugar; a super cookie is made up of butter cookie and a special ingredient.

When Pacman eats a butter cookie, she achieves 1 minute immunity from being consumed by a Ghost. When Pacman eats a super cookie, it takes a time lag of 30 seconds before she achieves 3 minutes of ghost-devouring power. (30 seconds is for the Ghost to run or devour the Pacman).

In the game, real Bluetooth-embedded objects are scattered in the game area to be collected as shown in Fig. 8. When the Pacman is within 10 meters of the Bluetooth object, communication takes place between the wearable computer and the Bluetooth device. After being alerted, she hunts for it in the surrounding physical area. Having found the object, collection is done simply by physically holding the object in her hands. Technically charge transfer sensing on the object (designed using QT161 IC chip from Quantum Research Group [16]) detects the player's touch and sends a message to the wearable computer which will send an corresponding message to the server to update the event. The collection of the ingredient will be kept in a virtual inventory list as seen in the figure as an icon. 
Collaboration between Players: There is an essential element of collaboration in the game play between a Pacman $\backslash$ Ghost with her Helper, and between any allied Pacmen.

(i) Pacman $\backslash$ Ghost and Helper Collaboration - The Helper always has a complete view of Pac-world, including the positions of all players and ingredients. Mobile players can also do so in VR mode, but AR mode is more advantageous for mobility. Furthermore Helpers collaborate among themselves to work out a strategy to achieve the team's goal. In this way, social interaction and collaboration is significant between Helpers, as well as between Helpers and her partner.

(ii) Pacman and Pacman Collaboration- Pacman players can collaborate through exchanging ingredients between them. For example Pacman A can initiates request for the list of unused ingredients Pacman B has. Upon approval, A can request for transfer of ingredient from B, subjected to approval by B. However, Pacman are not allowed to transfer special cookies so as not to disadvantage the Ghosts.

Use Special Cookie: All special cookies can only be used once. When a Pacman consumes a special cookie, an alert message is shown to her, and at the same time her Pacman avatar in the VR mode is labelled. This serves to inform all Helpers, including those from the Ghost-team, of her ability.

Devouring Enemy Player: To devour a Pacman, a Ghost must physically touch the Pacman's capacitive sensor pads on her shoulders as seen in Fig. 9. The same applies when a Pacman with Ghost-devouring capability devours a Ghost. When a Pacman player is the prey, her agility determines the "life-and-death" of her virtual Pacman role. Thus this computer game provides the benefits of natural wide area free bodily movements as part of humanistic interaction between each person.

Ending the Game: The game ends when either team meets their goal or when a time limit of ten minutes has been reached.

\subsection{Problems in Implementation}

From a broader perspective, the game of Human Pacman is a type of user adaptive application that is built upon the infrastructure of wearable and mobile computing, as well as the wireless multimedia communication. It aims to utilize the mentioned technology to provide nomadic players with personalized location based entertainment. However there are numerous problems associated with the actualization of these concepts.

We have identified three main problems in deploying the wireless communication network, in this case, the Wireless LAN of IEEE 802.11b. Firstly disconnections in communication often interrupt the flow of the game. Secondly limitation 
in bandwidth sets constraints on the type of multimedia data that can be sent between players and between the players and the server. For example we have to limit ourselves to simple text files for the frequent location, perspective, and status updates between the player's wearable computer and the server; and forego with the initial intention of sending live video streams between players. Thirdly unstable outdoor conditions often resulting in high error rate of the network. These three factors in turn increase communication latency which is due to retransmission, retransmission on time-out delays, error control processing, and short disconnections. We try to minimize the problems by carefully selecting the area for game play in the vicinity of the University campus in Singapore where network connectivity is good. Also, when designing the software for the game, we have embedded components that enable continual processing based on local data on the wearable computer so that when short disconnections occur, the game can still proceed without much disruptions.

Besides communication problems, we have to bear with constraints on the wearable computers too. Maintaining power for the computing device is essential in this system. Since Human Pacman is a game with short duration of play (recommended ten minutes), the wearable computer that is powered by two Sony Infolithium batteries lasting about three hours can adequately manage the task. Also, in designing for the user interface, we have considered the disadvantages of using HMD as mentioned by Duchamp at el [17] such as the hassle of the head gear, low-resolution, eye fatigue, and the requirement for dim lighting conditions. The problems mention also exist in Human Pacman since we are also using HMD for Augmented Reality outdoor gaming. Nevertheless as mentioned previously about the short duration of play in Human Pacman, the problem is so acute as to becoming unbearable to the players.

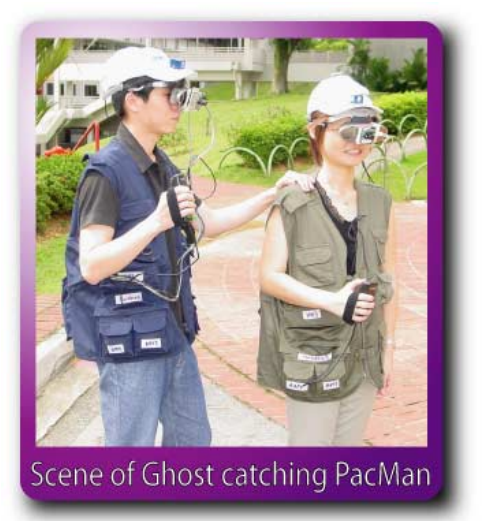

Fig. 9. A Ghost player tapping on the shoulder of Pacman player 


\section{Human Computer Interface Issues in Human Pacman}

Human Pacman envisions applying the concept of calm technology as described by Weiser [18] into computer gaming by experimenting with two of the interaction themes in Ubicomp, namely tangible interfaces and context-awareness. Details about them are discussed in the following subsection. After that, we will discuss Human Computer Interaction design issues in Human Pacman.

\subsection{Tangible Interface}

Even though Graphical User Interface (GUI) is still the dominant paradigm for interactions with computers, we are increasingly encountering computation that moves beyond the traditional confines of the desk and attempts to incorporate itself more richly into our daily experience of the physical and social world. Work on physical interaction started with the introduction of Computer-Augmented Environments [19] that have visioned the merging of electronic systems into the physical world.

Over the years, a number of projects have explored this new paradigm of interaction termed tangible computing. Early attempts include Bishop's Marble Answering Machine [20], "Brick" by Fitzmaurice [21], "Tangible Bits" and "mediaBlocks" from MIT media lab [22]. Nevertheless in all of these implementations of tangible computing, computer interaction remains passive with human initiating communication, and is confined between humans and virtual objects.

However, in Human Pacman, active communication and graspable interactions are explored with the use of embedded Bluetooth devices and capacitive sensors. Bluetooth is incorporated into the system where there is already Wireless LAN support because firstly it provides paired communication with security which is essential for one-to-one communication between the 'Ingredient' and the player; secondly Bluetooth devices support automatic device discovery and connection setup when they are within range therefore provide the backbone for reasoning by close physical proximity (Pacman has to search nearby area for 'Ingredient' once being alerted of it). Besides, tangible interaction between the Bluetooth embedded object and the player is made possible by using capacitive sensor for detecting the action of touch. Another important aspect is the clever exploitation of the affordances of the object's physical properties whereby players can intuitively associate the action of picking up the 'Ingredient' object with the collection of it in their virtual inventory.

The use of capacitive sensor shoulder pads of wearable computer for the detection of 'Devouring' action in game play, serves the purpose of demonstrating how computation can be used in concert with naturalistic activities. Also, by making the distinction between "interface" and "action" very much reduced, i.e. physical action of tapping versus a mouse-click for interaction, Human Pacman allows the players to experience transparent interchange between human and computer as never before in computer gaming. 


\subsection{Context Awareness in Outdoor Environment}

Researchers at Olivetti Research Ltd. (ORL) and Xerox PARC Laboratory pioneered the context-aware computing area with the introduction of Active Badge System and PARCTab [23][24]. However, these systems were expensive, and were confined to an indoor room. With the introduction of GPS and emergence of cheap but accurate sensors, a number of context-aware systems for outdoor applications were built. Notable systems include Georgia Tech Cyberguide project [25], and context-aware fieldwork tools at the University of Canterbury (an archeological assistant tool [26], a giraffe observation tool [27], and a rhino identification tool [28]). Unlike Human Pacman that uses augmented reality techniques as its main computer human interface, these systems have only primitive 2D maps and text presented on palmtops.

Another tourist assistant called Smart Sight was developed at the Carnegie Mellon University [29], which has an audio interface and aid navigational around the campus. Nevertheless since laptops were used as part of the mobile computer system, their weight and bulkiness have reduced user's mobility and comfort of use. In Human Pacman, players are provided with custom-built wearable computers.

There are three ways in which the idea of context awareness is being applied to in Human Pacman. Firstly, with the use of GPS and DRM, location awareness of players is made possible. Although GPS suffers from accuracy and selective availability, the problems are compensated through sensorfusion with DRM. The system's behavior is adapted to the player's current location for augmented reality (AR) placing of virtual cookies, and for calculating the relative positions of allied players.

Another important component in realizing AR elements in Human Pacman is the inertia sensor which provides the system with the current perspective of the player. Besides, Human Pacman also experiment with information context with the Helper player having information access to other players via Wireless LAN and providing them with necessary and timely information.

\subsection{Human Computer Interaction Design in Human Pacman}

In Human Pacman, we tried to combine materials from cognitive psychology and sociology with that from computer science. However the vast amount of issues encountered have exceeded the scope of this paper. Therefore we will concentrate on discussing issues with respect to Human Computer Interface design. According to Bellotti [30], there are five questions posing human-computer communication challenges for interaction design. In Table 2, we summarize the sensing approaches to interaction in Human Pacman with respect to the five questions raised.

\section{Conclusion}

The continual propagation of digital communication and entertainment in recent years forces many changes in societal psyche and lifestyle, i.e. how we think, 
Table 2. Five questions and answers posing human-computer communication challenges for interaction design in the case of Human Pacman

\begin{tabular}{|c|c|}
\hline Basic Question & Human Pacman Interface Answers \\
\hline $\begin{array}{l}\text { Address: } \\
\text { How do I address one } \\
\text { (or more) of many } \\
\text { possible devices? }\end{array}$ & $\begin{array}{l}\text { With the implementation of Ubiquitous Computing, the } \\
\text { system constitutes a more amorphous concept with auto- } \\
\text { mated interactions between sensors and computer. The } \\
\text { existence of unique address for each Bluetooth device } \\
\text { disambiguates the Bluetooth embedded objects. Further- } \\
\text { more, centralized control of the server prevents ambiguity } \\
\text { of intended target system even when there are more than } \\
\text { one players are near the Bluetooth device. Keyboard and } \\
\text { mouse are used for messaging and selection of the 'Ingre- } \\
\text { dients' to be exchanged between Pacmen. }\end{array}$ \\
\hline $\begin{array}{l}\text { Attention: } \\
\text { How do I know the } \\
\text { system is ready and } \\
\text { attending to my ac- } \\
\text { tions? }\end{array}$ & $\begin{array}{l}\text { Graphical feedback is used extensively from providing } \\
\text { alert message in popped up window, to refreshing virtual } \\
\text { inventory after Pacman picked up Bluetooth embedded } \\
\text { object. Also, since this graphical information is provided } \\
\text { in the HMD directly in the zone of the user's attention, } \\
\text { they are highly effective. }\end{array}$ \\
\hline $\begin{array}{l}\text { Action: } \\
\text { How do I effect } \\
\text { a meaningful action, } \\
\text { control its extent } \\
\text { and possibly specify } \\
\text { a target or targets } \\
\text { for my action? }\end{array}$ & $\begin{array}{l}\text { The Pacman } \backslash \text { Ghost click on preset messages to be sent } \\
\text { to Helpers. Pacmen click on graphical representation of } \\
\text { 'Ingredient' to be exchanged. Clearly labeled Bluetooth } \\
\text { embedded objects are to be found in physical space where } \\
\text { interaction is intuitive. According to Norman's Theory } \\
\text { of Action [31], this form of tangible interface bridges the } \\
\text { 'Gulf of Execution'. }\end{array}$ \\
\hline $\begin{array}{l}\text { Alignment: } \\
\text { how do I know the } \\
\text { system is doing } \\
\text { (has done) the right } \\
\text { thing? }\end{array}$ & $\begin{array}{l}\text { Real time graphical feedback presents distinctive and } \\
\text { timely graphical elements establishing the context of the } \\
\text { system. }\end{array}$ \\
\hline $\begin{array}{l}\text { Accident: } \\
\text { How do I avoid mis- } \\
\text { takes? }\end{array}$ & $\begin{array}{l}\text { Pacman right-click on virtual ingredient in order to dump } \\
\text { the ingredient. }\end{array}$ \\
\hline
\end{tabular}

work and play. With physical and mobile gaming gaining popularity, traditional paradigms of entertainment will irrevocably shake from the stale television-set inertia. We believe that Human Pacman heralds the conjuration and growth of a new genre of computer game that is built on mobility, physical actions and the real world as a playground. Reality, in this case, is becoming more exotic than fantasy because of the mixed reality element in the game play. On the other hand, emphasis on physical actions might even bring forth the evolvement 
of professional physical gaming as competitive sport of the future, for example 'PacMan International League'.

Element of social gaming in Human PacMan symbolizes the nas-cence of humanity in future digital entertainment. People are looking forward to widening their circle of friends and colleagues through social collaboration in game play. A new form of interactive entertainment is evolved.

Another important area of impact is the field of education. The technology presented in Human PacMan can be exported to applications in educational training that stresses on "learn by experience". Students are immersed in real site of action, and are given instructions visually through head mounted display or verbally through speaker $\backslash$ earphone. This technology serves as a powerful instrument of cognition since it can enhance both experimenting and reflective thoughts through mixed reality and interactive experience.

In conclusion, we believe Human PacMan is a pioneer in the new hybrid of physical, social, and mobile gaming that is built on ubiquitous computing and networking technology. The players are able to experience seamless transition between real and virtual world and therefore a higher than ever level of sensory gratification are obtained.

\section{References}

[1] Myers, D.: Computer game semiotics, Play and Culture. 1991, 4, 334-345. 209

[2] Weiser, M.: The computer for the 21st century. Scientific American 1991, 265(3), 94-100. 210

[3] Interactive Digital Software Association: State of the Industry - Report 2000-2001. [online document] 2001, Available at http://www.idsa.com. 210

[4] Interactive Digital Software Association: Essential Facts About the Computer and Video Game Industry. [online document] 2002, Available at http://www.idsa.com. 210

[5] Crawford, C.: Live: What a Concept!àNetworked Games. Digital Illusion, Dodsworth, C. Jr, ACM Press, 241-248. 210

[6] Björk, S., Falk, J., Hansson, R., and Ljungstrand, P.: Pirates! - Using the physical world as a game board. Interact 2001, IFIP TC. 13 Conference on HumanComputer Interaction, Tokyo, Japan 2001. 210

[7] Mandryk, R. L., and Inkpen, K. M.: Supporting free play in ubiquitous computer games. Workshop on Designing Ubiquitous Computer Games, UbiComp 2001, Atlanta, GA 2001. 210

[8] Oshima, T., Satoh, K., Yamamoto, H.,and Tamura, H.: AR2 Hockey system: A collaboration mixed reality system. Trans VRSJ 1998; 3(2); 55-60. 210

[9] Tamura, H., Yamamoto, H.,and Katayama, A.: Mixed Reality: Future Dreams Seen at the Border between Real and Virtual Worlds. Computer Graphics and Applications, vol. 21, no. 6 pp. 64-70, 2001. 210

[10] Thomas, B., Close, B., Donoghue, J., Squires, J., Bondi, P. D.,and Piekarski, W.: First Person Indoor $\backslash$ Outdoor Augmented Reality Application: ARQuake. Personal and Ubiquitous Computing, vol. 6, no. 1, pp 75-86, 2002. 210

[11] Milgram, P.,and Kishino, F.: A Taxanomy of Mixed Reality Visual Displays. IECE Trans on Information and Systems (Special Issue on Networked Reality), vol E77D, no. 12, 1321-1329, 1994. 210 
[12] Billinghurst, M., Campbell, S., Hendrickson, D., Chinthammit, W., Poupyrev, I., Takahashi, K.,and Kato, H.: Magic Book: Exploring Transitions in Collaborative AR Interfaces. SIGGRAPH 2000, 2000. 210

[13] Cheok, A. D., Yang, X., Zhou, Z. Y., Billinghurst, M., and Kato, H.: Touch-Space: Mixed Reality Game Space Based on Ubiquitous, Tangible, and Social Computing. Personal and Ubiquitous Computing, vol. 6, no. 2, 430-442, 2002. 210

[14] InterSense Inc: Inertia Cube2 Bringing 3D To Life - Manual for Serial Port Model, 2001. 213

[15] Point Grey Research Corporation, http://www.ptgrey.com. 213

[16] Quantum Research Group Ltd, [online document] 2002. Available at http://www. qprox.com. 216

[17] Duchamp, D., Steven, K. F., and Gerald Jr. Q. M., "Software Technology for Wireless Mobile Computing", IEEE Network Magazine, 12-18, Nov 1991. 218

[18] Weiser, M.: Some computer science issues in ubiquitous computing. Communications of ACM, 36, 7: Special issue on computer augmented environments: back to the real world. 219

[19] Computer Augmented Environments: Back to the Real World. Communications of the ACM, July 1993, 36, 7. 219

[20] Smith, C. G.: The Hand That Rocks the Cradle. I. D., May/June 1995, 60-65. 219

[21] Fitzmaurice, G., et al.: Bricks: Laying the Foundations for Graspable User Interfaces. Proceedings of the Conference on Human Factors in Computing Systems (CHI '95), ACM, Denver, May 1995, 442-449. 219

[22] Ishii, H., and Ullmer, B.: Tangible Bits: Towards Seamless Interfaces between People, Bits, and Atoms. Proceedings of the Conference on Human Factors in Computing Systems (CHI '97), March 22-27, 1997. 219

[23] Want, R., Hopper, A., Falco, V.,and Gibbons, J.: The Active Badge location system. ACM Transactions on Information Systems, 10(1):91-102, January 1992. 220

[24] Schilit, B. N., Theimer, M. M.,and Welch, B. B.: Customizing mobile applications. In Proceedings of USENIX Mobile \& Location-Independent Computing Symposium, Cambridge, Massachusetts, August 1993. USENIX Association, 129-138. 220

[25] Long, S., et al: Rapid Prototyping of Mobile Context-aware Applications: The Cyberguide Case Study. 2nd ACM International Conference on Mobile Computing and Networking (MobiCom'96) 1996 November 10-12, 1996. 220

[26] Ryan, N., Pascoe, J.,and Morse, D.: Enhanced Reality Fieldwork: the ContextAware Archaeological Assistant. Gaffney, V., Van Leusen, M., Exxon, S., Eds. Computer Applications in Archaeology.

http://www.cs.ukc.ac.uk/pubs/1998/616/content.html. 220

[27] Pascoe, J., Ryan, N.S., and Morse, D. R.: Human Computer Giraffe Interaction HCI in the Field. Workshop on Human Computer Interaction with Mobile Devices. http://www.dcs.gla.ac.uk/ johnson/papers/mobile/HCIMD1.html. 220

[28] Pascoe, J., Ryan, N.S.,and Morse, D. R.: Issues in Developing Context-Aware Computing. Proceedings of the International Symposium on Handheld and Ubiquitous Computing, Karlsruhe, Germany, September 1999, Springer-Verlag, 208221. 220

[29] Yang, J., Yang, W., Denecke, M.,and Waibel, A.: Smart sight: a tourist assistant system. 3rd International Symposium on Wearable Computers, San Francisco, California, 18-19 October, 1999, 73-78. 220 
[30] Bellotti, V.M.E., Back, M. J., Edwards, W.K., Grinter, R.E., Lopes, C. V.,and Henderson, A.: Making sense of sensing systems: Five questions for designers and researchers. Proc. CHI 2002, 415-422. ACM. 220

[31] Norman, D. A.: The Design of Everyday Things. Doubleday: New York, New York. 221 